\title{
Euclidean Invariance in Statistical Mechanics of Classical Continuous System
}

\author{
Ch. Gruber, Ph. A. Martin, and Ch. Oguey \\ Institut de Physique Théorique, CH-1000 Lausanne, Switzerland
}

\begin{abstract}
It is shown that equilibrium states of classical particles with short range interactions are Euclidean invariant whenever their correlation functions have a clustering which is integrable. The relation between invariance and clustering is analysed for spatial rotations and internal rotational degrees of freedom. The analysis is then extended to the case of long range interactions, including the Coulomb force and jellium systems.
\end{abstract}

\section{Introduction}

An equilibrium state which spontaneously breaks a continuous symmetry cannot have an exponentially fast clustering: this is a general formulation of the Goldstone theorem in statistical mechanics. However, to analyse the possible existence of crystalline phases or the existence of phases with orientational order, a more precise formulation of the Goldstone theorem is necessary.

In [1] we have proved a version of the Goldstone theorem for the translation group, namely $\mathscr{L}^{1}$-clustering states are necessarily invariant under translation. In the present paper, we shall be more specifically concerned with the rotation group; we shall also improve some of the previous results concerning systems with short range interactions. Furthermore, in addition to spatial rotations, we shall also consider systems of particles with internal degrees of freedom, for example classical gas of anisotropic molecules.

We treat separately the case of short range forces (Sect. II) and that of long range forces (Sect. III). In the latter case, which includes $N$-component plasma and jellium systems (i.e. charged particles with a rigid uniform neutralizing background), the main idea is that the effect of the long range can be taken care by means of sum rules which reflect the shielding property of such systems. $[4,12]$.

Our definition of equilibrium states and the starting point of our investigation is the BBGKY-hierarchy for the correlation functions. For sufficiently well behaved short range forces it is known that the BBGKY-hierarchy is equivalent to other definitions of equilibrium states such as the classical KMS condition, the Dobrushin-Lanford-Ruelle or the Kirkwood-Salzburg equations. (See [2] and 
the references quoted there for instance.) Furthermore, all those equations are verified by the states obtained as the thermodynamic limit of finite volume Gibbs distributions (for a direct proof of this fact in relation with BBGKY-equation, see [3]). On the other hand, for systems with long range forces, it is known that equilibrium states can stili be defined by means of a BBGKY-equation [4]. For this reason, we have taken the BBGKY formalism for our discussion.

The relations which we shall derive between clustering and symmetry breaking, imply that in dimension $v \geqq 2$ a phase with spontaneous symmetry breakdown must have a clustering which can not decay faster than $|x|^{-\delta}$ where $\delta$ is specified in the Table 1:

Table 1

\begin{tabular}{llll}
\hline & $\begin{array}{l}\text { Short range } \\
\text { Force }\end{array}$ & $\begin{array}{l}\text { N-Component } \\
\text { Coulomb Systems } \\
\text { (without background) }\end{array}$ & $\begin{array}{l}\text { Jellium } \\
\text { Systems }\end{array}$ \\
\hline Translation & $v-1$ & $v+1$ & $v+1$ \\
Internal rotations & $v-1$ & $v+1$ & $v+1$ \\
Spatial rotations & $v$ & $v+1$ & $v+2$ \\
\hline
\end{tabular}

The values of $\delta$ obtained in our proof may not be optimal. Indeed, a recent study of the planar rotator model reveals that the two point correlation function decays exactly as $\frac{1}{|x|}$ when $v=3[5]$ and similar behaviour is expected in the case of the 3-dimensional anharmonic crystal [5-7]. Moreover, for short range forces, there does not exist any equilibrium state which breaks the translation or internal rotation in two dimensions $[8,9]$. This result leads us to conjecture that for these symmetries the optimal value of $\delta$ could be $v-2$ instead of $v-1$.

In fact it can be shown [14] with the Bogoliubov inequality that any crystalline phase must have a clustering slower or equal to $|x|^{-(\nu-2)}$. However, the question remains open for other inhomogeneous situations such as the interface.

Several papers concerning the Goldstone theorem for short range forces have appeared recently. The same result as ours on the breaking of translation invariance was obtained in $[15,16]$ on the basis of the KMS condition: an inhomogeneous phase has a clustering slower or equal to $|x|^{-(v-1)}$. Using the Bogoliubov inequality, quantum lattice systems are treated in $[10,14]$ with similar results. Continuous quantum systems briefly discussed in [10], are under current study and will be presented in forthcoming publications.

\section{Definition of the System}

The system consists of particles in $\mathbb{R}^{v}$ having internal degrees of freedom. The internal degrees of freedom are labelled by the points $\omega$ of some (locally compact, Hausdorff) measurable space $\Omega$ with finite measure. We may think of a particle as a rotator with $\omega$ a unit vector defining its axis; in this case $\Omega=S^{(v)}$ is the unit sphere in $\mathbb{R}^{v}$ and $d \omega$ is the invariant measure on $S^{(v)}$. We could also think of a 
particle as a molecule and $\omega$ would label the specie and the relative positions of the atoms inside the molecule.

We shall assume that there is a representation of the rotation group $S O(v)$ on $\Omega$ which leaves the measure $d \omega$ invariant. We denote $\Gamma=\mathbb{R}^{v} \times \Omega$ the configuration space; $q=(x, \omega), x \in \mathbb{R}^{v}, \omega \in \Omega$ will denote a point in $\Gamma$ and $\int d q=\int_{\mathbb{R}^{\prime}} d x \int_{\Omega} d \omega$.

We introduce furthermore $\nabla$ and $J$ the generators of the translation and rotation groups acting on differentiable functions $f(q)$ on $\Gamma$; we then have:

$$
J=L+S,
$$

where $L$ and $S$ are the generators of the rotation group acting respectively on $\mathbb{R}^{v}$ and $\Omega$.

The particles interact by means of two-body potentials $\Phi\left(q_{1}, q_{2}\right)$ which we require to be symmetric under permutations, Euclidean invariant and regular. The symmetry and invariance conditions are expressed by:

$$
\left.\begin{array}{l}
\Phi\left(q_{1}, q_{2}\right)=\Phi\left(q_{2}, q_{1}\right), \\
\Phi\left(q_{1}, q_{2}\right)=\Phi\left(x_{1}-x_{2}, \omega_{1}, \omega_{2}\right), \\
\Phi\left(q_{1} ; q_{2}\right)=\Phi\left(R\left(x_{1}-x_{2}\right), R \omega_{1}, R \omega_{2}\right), \quad \forall R \in S O(v) .
\end{array}\right\}
$$

The regularity is the condition that the force $F\left(q_{1}, q_{2}\right)=F\left(x_{1}-x_{2}, \omega_{1}, \omega_{2}\right)=-\nabla_{1}$ $\Phi\left(q_{1}, q_{2}\right)$ and $J_{1} \Phi\left(q_{1}, q_{2}\right)$ be continuous for $x_{1} \neq x_{2}{ }^{1}$; the Euclidean invariance of the potential is then expressed by:

$$
\left(\nabla_{1}+\nabla_{2}\right) \Phi\left(q_{1}, q_{2}\right)=0 ; \quad\left(J_{1}+J_{2}\right) \Phi\left(q_{1}, q_{2}\right)=0 .
$$

Combining the symmetry and the Euclidean invariance yields

$$
\nabla_{1} \Phi\left(q_{1}, q_{2}\right)=-\nabla_{2} \Phi\left(q_{2}, q_{1}\right) ; \quad\left(J_{1} \Phi\right)\left(q_{1}, q_{2}\right)=-J_{2} \Phi\left(q_{2}, q_{1}\right) .
$$

The states $\rho$ of the system are described by the set of correlation functions $\rho\left(q_{1}, \ldots, q_{n}\right)=\rho(Q), n=1,2, \ldots$ They are symmetric functions of $\left(q_{1}, \ldots, q_{n}\right)$ and are assumed to satisfy the following regularity condition:

$\rho\left(q_{1}, \ldots, q_{n}\right)$ is continuous, uniformly bounded on $\Gamma^{n} ; \nabla_{1} \rho$ and $J_{1} \rho$ are locally $\mathscr{L}^{1}$ and continuous in any open set which does not contain coincident particles.

Throughout the paper we shall always assume that the states are clustering in the following sense:

$$
\left.\begin{array}{l}
\rho^{T}\left(q_{1}, \ldots, q_{n}\right)=O\left(\frac{1}{\left|x_{1}\right|^{\delta}}\right) \text { as }\left|x_{1}\right| \rightarrow \infty \\
\text { for } \omega_{1}, q_{2}, \ldots, q_{n} \text { fixed, } n=2,3, \ldots \\
\text { uniformly with respect to } \omega_{1} \text { and } \\
\text { uniformly with respect to } q_{2} \text { for } n \geqq 3 .
\end{array}\right\}
$$

1 With the notation $\nabla_{j}, J_{j}$ generators of the Euclidean group on the space of functions $f\left(q_{j}\right)$ 
$\rho^{T}(Q)$ are the truncated correlation functions defined in the usual way and $\delta$ a positive number to be defined later.

Let us recall that a state $\rho$ is:

invariant under translations

$$
\text { if } \sum_{i=1}^{n} \nabla_{i} \rho=0
$$

invariant under rotations

$$
\text { if } \sum_{i=1}^{n} J_{i} \rho=0
$$

invariant under spatial rotations if $\sum_{i=1}^{n} L_{\imath} \rho=0$,

invariant under internal rotations if $\sum_{i=1}^{n} S_{i} \rho=0$.

\section{Systems with Short Range Forces}

In this section, we shall consider the case of short-range forces, i.e. we shall assume that the force $F\left(q_{1}, q_{2}\right)=-\nabla_{1} \Phi\left(q_{1}, q_{2}\right)$ and $J_{1} \Phi\left(q_{1}, q_{2}\right)$ are integrable over $\mathbb{R}^{v} \times \Omega \times \Omega$ :

$$
\begin{gathered}
\int_{\mathbb{R}^{\nu}} d x \int_{\Omega} d \omega_{1} \int_{\Omega} d \omega_{2}\left|F\left(x, \omega_{1}, \omega_{2}\right)\right|<\infty, \\
\int_{\mathbb{R}^{\nu}} d x \int_{\Omega} d \omega_{1} \int_{\Omega} d \omega_{2}\left|J \Phi\left(x, \omega_{1}, \omega_{2}\right)\right|<\infty .
\end{gathered}
$$

Following the usual derivation of the BBGKY-Equations, the Equilibrium states will be defined by correlation functions which are a solution of the following hierarchy of equations:

$$
-k T G_{1} \rho\left(q_{1}, Q\right)=\sum_{j=2}^{n}\left(G_{1} \Phi\right)\left(q_{1}, q_{j}\right) \rho\left(q_{1}, Q\right)+\int d q\left(G_{1} \Phi\right)\left(q_{1}, q\right) \rho\left(q_{1}, q, Q\right),
$$

where $Q=\left(q_{2}, q_{3}, \ldots, q_{n}\right)$ if $n=2,3, \ldots, Q=\varnothing$ if $n=1$, and $G$ denotes either of the generators $\nabla, L, S$ or $J$.

The main result of this section, i.e. any $\mathscr{L}^{1}$ clustering equilibrium state is necessarily invariant under rotations, is stated in the following proposition.

Proposition 1. Let $T \neq 0$; if $\Phi\left(q_{1}, q_{2}\right)$ satisfies Eq. (7) and

$$
\int_{\mathbb{R}^{v}} d x \int_{\Omega} d \omega_{1} \int_{\Omega} d \omega_{2}|x|^{v+1}\left|F\left(x, \omega_{1}, \omega_{2}\right)\right|<\infty,
$$

then any equilibrium state which satisfies the clustering condition Eq. (6) with $\delta>v$ is rotation invariant.

Proof. Using the BBGKY-Equations (8) together with Eq. (4), the equilibrium states will be solution of the following equation:

$$
-k T \sum_{j=2}^{n} J_{j} \rho(Q)=\int d q_{1}\left[\sum_{j=2}^{n}\left(J_{j} \Phi\right)\left(q_{j}, q_{1}\right)\right] \rho\left(q_{1}, Q\right), Q=\left(q_{2}, \ldots, q_{n}\right) .
$$


On the other hand, Eq. (8) with $Q=\left(q_{2}, \ldots, q_{n}\right)$ yields:

$$
\sum_{j=2}^{n}\left(J_{1} \Phi\right)\left(q_{1}, q_{j}\right) \rho\left(q_{1}, Q\right)=-k T J_{1} \rho\left(q_{1}, Q\right)-\int d q\left(J_{1} \Phi\right)\left(q_{1}, q\right) \rho\left(q_{1}, q, Q\right) .
$$

We then integrate both sides of Eq. (11) on $q_{1}$ with $x_{1}$ restricted to a ball of radius $R$ centered at the origin.

The integral on the left hand side of Eq. (11) exists in the limit $R \rightarrow \infty$ by the integrability condition Eq. (7). Using Eq. (5) together with Eq. (10) and (11), we obtain:

$$
\begin{gathered}
-\sum_{j=2}^{n} J_{j} \rho(Q)=\lim _{R \rightarrow \infty} \int_{\left|x_{1}\right| \leqq R} d q_{1}\left[\left(J_{1} \rho\right)\left(q_{1}, Q\right)+\beta \int d q\left(J_{1} \Phi\right)\left(q_{1}, q\right)\right. \\
\left.\rho\left(q_{1}, q, Q\right)\right], \quad Q=\left(q_{2}, \ldots, q_{n}\right) .
\end{gathered}
$$

We shall prove that the right hand side Eq. (12) vanishes in the limit $R \rightarrow \infty$, which will thus establish the invariance of the state under rotations. In order to use the clustering condition on the state, it is convenient to use the first equation of the BBGKY hierarchy Eq. (8) to express the right hand side of Eq. (12) in the form:

$$
\begin{aligned}
\left(\sum_{j=2}^{n} J_{j} \rho\right)(Q)= & -\lim _{R \rightarrow \infty} \int_{\left|x_{1}\right| \leqq R} d q_{1} J_{1}\left(\rho\left(q_{1}, Q\right)-\rho\left(q_{1}\right) \rho(Q)\right) \\
& -\beta \lim _{R \rightarrow \infty} \int_{\left|x_{1}\right| \leqq R} d q_{1} \int d q\left(J_{1} \Phi\right)\left(q_{1}, q\right)\left(\rho\left(q_{1}, q, Q\right)-\rho\left(q_{1}, q\right) \rho(Q)\right) .
\end{aligned}
$$

The limit of both terms in Eq. (13) exists and is zero. Indeed: let $h\left(q_{1}\right)=\rho\left(q_{1} Q\right)-$ $\rho\left(q_{1}\right) \rho(Q)$; the first term on the right hand side of Eq. (13) becomes

$$
\lim _{R \rightarrow \infty} \int_{\left|x_{1}\right| \leqq R} d q_{1}\left(L_{1}+S_{1}\right) h\left(q_{1}\right)=\lim _{R \rightarrow \infty} \int_{\left|x_{1}\right| \leqq R} d x_{1} \int_{\Omega} d \omega_{1}\left(L_{1}+S_{1}\right) h\left(x_{1}, \omega_{1}\right)
$$

and the clustering condition Eq. (6) implies $h\left(x_{1} \omega_{1}\right)=O\left(\frac{1}{\left|x_{1}\right|^{\delta}}\right)$ uniformly with respect to $\omega_{1}$. Using furthermore the regularity condition of $\rho$, we have

$$
\begin{aligned}
\int_{\left|x_{1}\right| \leqq R} d x_{1}\left(L_{1} h\right)\left(q_{1}, Q\right)= & \int_{\left|x_{1}\right| \leqq R} d x_{1}\left(x_{1} \wedge \nabla_{1}\right) h\left(x_{1}, \omega_{1}\right) \\
= & \int_{\left|x_{1}\right|=R} x_{1} \wedge d \sigma_{1} h\left(x_{1}, \omega_{1}\right)=O\left(\frac{1}{R^{\delta-v}}\right) \\
& \text { as } R \rightarrow \infty
\end{aligned}
$$

uniformly with respect to $\omega_{1}$.

On the other hand since $\Omega$ has finite measure and $d \omega$ is invariant under the rotation group, the regularity condition of the state implies that

$$
\int_{\left|x_{1}\right| \leqq R} d x_{1} \int_{\Omega} d \omega_{1}\left(S_{1} h\right)\left(x_{1}, \omega_{1}\right)=0 .
$$

This identity follows from the following consideration; let $S$ be the generator of the rotation around a given axis $\hat{n}$ and let $\tau_{\varphi}$ denote the action of a rotation of angle $\varphi$ around $\hat{n}$ on $\Gamma$; then 


$$
\begin{aligned}
\int_{\left|x_{1}\right| \leqq R} d x_{1} \int_{\Omega} d \omega_{1}\left(S_{1} h\right)\left(q_{1}\right)= & \frac{1}{2 \pi} \int_{0}^{2 \pi} d \varphi \int_{\left|x_{1}\right| \leqq R} d x_{1} \int_{\Omega} d \omega_{1}\left(S_{1} h\right)\left(\tau_{\varphi} q_{1}\right) \\
= & \int_{\left|x_{1}\right| \leqq R} d x_{1} \int_{\Omega} d \omega_{1} \frac{1}{2 \pi} \int_{0}^{2 \pi} d \varphi \lim \frac{1}{\alpha} \\
& \cdot\left[h\left(\tau_{\alpha} \tau_{\varphi} q_{1}\right)-h\left(\tau_{\varphi} q_{1}\right)\right] \\
= & \int_{\left|x_{1}\right| \leqq R} d x_{1} \int_{\Omega} d \omega_{1} \frac{1}{2 \pi} \int_{0}^{2 \pi} d \varphi \frac{d}{d \varphi} \psi(\varphi)=0,
\end{aligned}
$$

where $\psi(\varphi)=h\left(\tau_{\varphi} q_{1}\right)$.

We have thus shown that the first term on the right hand side of Eq. (13) is zero; using Eq. (4) the second term can be written as

$$
\frac{1}{2} \int_{\left|x_{1}\right| \leqq R} d q_{1} \int d q\left(J_{1}-J\right) \Phi\left(q_{1}, q\right)\left[\rho\left(q_{1}, q, Q\right)-\rho\left(q_{1}, q\right) \rho(Q)\right] .
$$

To show that (16) vanishes in the limit $R \rightarrow \infty$ we note that

$$
\begin{aligned}
\rho\left(q_{1}, q, Q\right)-\rho\left(q_{1}, q\right) \rho(Q)= & \rho\left(q_{1}\right)(\rho(q, Q)-\rho(q) \rho(Q)) \\
& +\rho(q)\left(\rho\left(q_{1}, Q\right)-\rho\left(q_{1}\right) \rho(Q)\right)+R\left(q_{1}, q, Q\right),
\end{aligned}
$$

where $R\left(q_{1}, q, Q\right)$ is a sum of products of truncated functions where the arguments $q$ or $q_{1}$ (or the pair $\left(q_{1}, q\right)$ ) always occur in conjunction with some other argument $q_{i} \in Q, i=2, \ldots n$. (See Lemma 4, p. 219 [4].) Therefore, the clustering condition Eq. (6) implies that

$$
\left|\rho\left(q_{1}, q, Q\right)-\rho\left(q_{1}, q\right) \rho(Q)\right| \leqq \frac{M_{1}}{\left|x_{1}\right|^{\delta}}+\frac{M_{2}}{|x|^{\delta}}
$$

with $M_{1}, M_{2}$ independent of $q_{1}$ and $q$.

Let us write

$$
g\left(q_{1}, q\right)=\rho\left(q_{1}, q, Q\right)-\rho\left(q_{1}, q\right) \rho(Q)
$$

and consider first the contribution of internal rotations $\left(S_{1}-S\right) \Phi$ to Eq. (16). It follows from (7) and (18) that

$$
\left(S_{1}-S\right) \Phi\left(q_{1}, q\right) g\left(q_{1}, q\right)
$$

is integrable over $\Gamma^{2}$ so that we can take the limit $R \rightarrow \infty$ to obtain:

$$
\frac{1}{2} \int d q_{1} \int d q\left(S_{1}-S\right) \Phi\left(q_{1}, q\right)\left[\rho\left(q_{1}, q, Q\right)-\rho\left(q_{1}, q\right) \rho(Q)\right]=0
$$

because of the symmetry of the correlation functions and the antisymmetry of $\left(S_{1}-S\right) \Phi\left(q_{1}, q\right)$.

Finally, we show in the Appendix that the contribution of spatial rotations $\left(L_{1}-L\right) \Phi$ to Eq. (16) vanishes in the limit $R \rightarrow \infty$ which concludes the proof of Proposition 1.

The translation invariance of the state has been established in [1] when there are no internal degrees of freedom.

By obvious modifications of the present proof (i.e. dealing with the generator 
of translation $\nabla$ instead of $J$ ), we obtain

Proposition 2. Let $T \neq 0$; if $\Phi\left(q_{1}, q_{2}\right)$ satisfies Eq. (7) and

$$
\int_{\mathbb{R}^{v}} d x \int_{\Omega} d \omega_{1} \int_{\Omega} d \omega_{2}|x|^{v}\left|F\left(x, \omega_{1}, \omega_{2}\right)\right|<\infty,
$$

then any equilibrium state satisfying the clustering condition (6) with $\delta>v-1$ is translation invariant.

Corollary 1. Any equilibrium state satisfying the conditions of Prop. 1 is invariant under the full Euclidean group.

Remarks. 1) For short range interactions any equilibrium state which breaks the translation invariance must have a clustering which decays slower or as $|x|^{-v+1}$ whereas to break the rotation invariance we need only to have a clustering slower or equal to $|x|^{-\nu}$.

The reason for this difference is that, $L=x \wedge \nabla$ being linear in $x$, one more power in the decay of correlation functions is needed to control the convergence of integrals (see the Appendix, in particular (A4)).

This indicates that in the transition from a Euclidean invariant state to a state of lower symmetry, rotation invariance is likely to be broken before translation invariance.

2) Two dimensional systems are of particular interest in this respect: they are known to be translation invariant irrespective of any clustering condition (for short range forces) [9]. However, they may have phases noninvariant under spatial rotations with clustering decaying slower or as $|x|^{-2}$.

3) If the force satisfies only the integrability condition (7) (but has no finite moments as assumed in (9)), then the rotation invariance (respectively the translation invariance) can still be proved provided that the clustering (6) holds with $\delta>v+1$ (respectively $\delta>v$ ). Indeed, in this case, the integrand of the expression (A1) in the Appendix is integrable in $q_{1}$ and $q$ over $\Gamma \times \Gamma$ and antisymmetric, showing that the integral (A1) exists and vanishes.

4) The same result will also hold if the force has a nonintegrable repulsive singularity at $x=0$. Then, the correlation function should vanish at coincident points in such a way that $\rho\left(q_{1}, q_{2}, Q\right) F\left(q_{1}, q_{2}\right)$ is still integrable in $q_{1}=q_{2}$ (at finite volume, this follows from an integrability condition on $\exp \left(-\beta \Phi\left(q_{1}, q_{2}\right)\right) F\left(q_{1} q_{2}\right)$ in $q_{1}=q_{2}$ ). In this case, the additional assumption (supplementing (18)) is therefore natural

$$
\left|\rho\left(q_{1}, q_{2}, Q\right)-\rho\left(q_{1}, q_{2}\right) \rho(Q)\right| \leqq \zeta\left(x_{1}-x_{2}\right)\left(\frac{M}{\left|x_{1}\right|^{\delta}}+\frac{M}{\left|x_{2}\right|^{\delta}}\right)
$$

with $M$ independent of $x_{1}, \omega_{1}, x_{2}, \omega_{2}$ and $\zeta(x)$ is a bounded function such that $\zeta(x) F\left(x, \omega_{1}, \omega_{2}\right)$ is locally integrable in $x=0$. It is then easy to check that the proofs of Prop. 1 and Prop. 2 can be carried out in the same way. Finally, the case of strict hard cores can be treated with the same results [11].

If the potential is separately invariant under space rotations and (or) internal 
rotations, i.e.

$$
\left(L_{1}+L_{2}\right) \Phi\left(q_{1}, q_{2}\right)=0
$$

and (or)

$$
\left(S_{1}+S_{2}\right) \Phi\left(q_{1}, q_{2}\right)
$$

we deduce the corresponding invariance properties of the states as corollaries of Proposition 1, i.e.

$$
\left(\sum_{i=1}^{n} L_{i}\right) \rho(Q)=0
$$

and (or)

$$
\left(\sum_{i=1}^{n} S_{i}\right) \rho(Q)=0 .
$$

Corollary 2. Let $\Phi\left(q_{1}, q_{2}\right)$ be invariant under spatial rotations. Then any equilibrium state satisfying the conditions of Proposition 1 is also invariant under spatial rotations.

Corollary 3. Let $\Phi\left(q_{1}, q_{2}\right)$ be invariant under internal rotations and verify

$$
\int_{\Omega} d \omega_{1} \int_{\Omega} d \omega_{2} \int_{\mathbb{R}^{v}} d x|x|^{v}\left|S_{1} \Phi\left(x, \omega_{1}, \omega_{2}\right)\right|<\infty .
$$

Then, any equilibrium state satisfying the clustering condition (6) with $\delta>v-1$ is invariant under internal rotations.

Corollary 2 follows immediately when one deals only with the generator of spatial rotations in Prop. 1.

Corollary 3 is proven as Proposition 1 with the antisymmetric function $\left(S_{1}-S\right)$ $\Phi\left(q_{1}, q\right)$ replacing $\left(L_{1}-L\right) \Phi\left(q_{1} q\right)$ in the estimates given in the Appendix (here a clustering which is $O\left(\frac{1}{|x|^{\delta}}\right), \delta>v-1$ is again sufficient since $S$ does not introduce an additional power of $x$ ).

Remarks. 1) If the potential satisfies the integrability condition (7) (but not (25)), the result of Corollary 3 is still true provided that the clustering (6) holds with $\delta>v$ (see Remark 3 following Corollary 1).

2) All these results can be generalized to the case where the internal degree of freedom consists in the elements of an arbitrary compact Lie group. Indeed, besides the integrability and clustering hypothesis, one uses only the fact that the internal symmetry group has generators acting in differential form and an invariant measure with finite total mass.

\section{Systems with Long Range Forces}

In this section, we consider systems which consist of $N$ species of "charged" particles and we denote by $\sigma_{\alpha}, \alpha=1, \ldots, N$ the charge of the specie $\alpha$. To simplify the following discussion we consider point particles without internal degrees of freedom other than the charge. We shall then add a few remarks at the end of this section 
concerning the extension to systems of particles with internal degrees of freedom. We denote by $q=(x, \alpha) \in \mathbb{R}^{v} \times\{1, \ldots, N\}$ the position and specie of the particle and $\int d q=\int d x \sum_{\alpha}$

The particles interact by means of a two-body potential of the form

$$
\Phi\left(q_{1}, q_{2}\right)=\sigma_{\alpha_{1}} \sigma_{\alpha_{2}} \Phi\left(\left|x_{1}-x_{2}\right|\right)
$$

where $\Phi(x)$ is of class $C^{3}$ on $\mathbb{R}^{v}$. The long range of the interaction is characterized by the asymptotic behaviour of the force $F(x)=-\nabla \Phi(x)$ as $|x| \rightarrow \infty$ :

$$
\begin{gathered}
F(x)=\frac{\hat{x}}{|x|^{\gamma}}+o\left(\frac{1}{|x|^{\gamma}}\right), \quad \hat{x}=\frac{x}{|x|^{\prime}}, \quad|x| \rightarrow \infty, \\
\frac{\partial}{\partial x^{r}} F(x)=O\left(\frac{1}{|x|^{\gamma+1}}\right), \quad \frac{\partial^{2}}{\partial x^{r} \partial x^{s}} F(x)=O\left(\frac{1}{|x|^{\gamma+2}}\right) .
\end{gathered}
$$

The case of main interest is the Coulomb force corresponding to $\gamma=v-1$; another case of interest is the system of electrons confined to a narrow layer above the surface of liquid helium which corresponds to $\gamma=v=2$. In the following, we consider general values of $\gamma$ such that $v-1 \leqq \gamma \leqq v$ and we recall the notation:

$$
F\left(q_{1}, q_{2}\right)=\sigma_{\alpha_{1}} \sigma_{\alpha_{2}} F\left(x_{1}-x_{2}\right)
$$

The equilibrium states are now defined by their correlation functions which are clustering solutions of the BBGKY hierarchy written in the following manner:

$$
\begin{aligned}
k T \nabla_{1} \rho\left(q_{1}, Q\right)= & {\left[\sigma_{\alpha_{1}} E_{\rho}\left(x_{1}\right)+\sum_{j=2}^{n} F\left(q_{1}, q_{j}\right)\right] \rho\left(q_{1}, Q\right) } \\
& +\int d q F\left(q_{1}, q\right)\left[\rho\left(q_{1} q Q\right)-\rho(q) \rho\left(q_{1}, Q\right)\right],
\end{aligned}
$$

where $Q=\left(q_{2}, \ldots, q_{n}\right)$ if $n \geqq 2, Q=\varnothing$ if $n=1$,

$$
E_{\rho}(x)=E_{0}+\int d y(F(x-y)-F(-y)) c_{\rho}(y)
$$

is the average electric field in the state $\rho$ and $c_{\rho}(x)=\sum_{\alpha=1}^{N} \sigma_{\alpha} \rho_{\alpha}(x)-\rho_{B}$ is the average charge density.

$E_{0}=E_{\rho}(0)$ and $\rho_{B}$ are parameters describing the systems. Systems with $\rho_{B} \neq 0$ are called "Jellium"; they correspond to systems of charged particles in the presence of a rigid, uniformly charged background, with charge density $-\rho_{B}$.

In the case of Coulomb systems $(\gamma=v-1)$ we consider only states which are invariant under some discrete subgroup of the translation group; this condition on the equilibrium state will then imply that the state is locally neutral and that the field $E_{\rho}(x)$ Eq. (29) is well defined.

We refer to $[2,4]$ for a discussion of Eq. (28) as the definition of equilibrium states for systems with long range forces.

Proposition 3. Let $T>0, v \geqq 2$ and assume that $\Phi\left(q_{1}, q_{2}\right)$ verifies Eq.(26),(27) Then i) any equilibrium state satisfying the clustering condition Eq. (6) with $\delta>v+2$ 
and

$$
\int_{\mathbb{R}^{v}} d x_{1} \int_{\mathbb{R}^{v}} d x_{2}\left|x_{1}\right|\left|\rho_{\alpha_{1}, \ldots, \alpha_{n}}^{T}\left(x_{1}, \ldots, x_{n}\right)\right|<\infty \quad \forall n \geqq 3
$$

is invariant under rotation.

ii) for $N$ component Coulomb systems with $\rho_{B}=0$ the same result will also be valid if the equilibrium state satisfies the clustering condition with $\delta>v+1$.

Corollary. Any equilibrium state satisfying the conditions of Prop. 3 is invariant under the full Euclidean group.

Indeed it was already shown in [1] that a state verifying the clustering condition with $\delta>v+1$ is necessarily translation invariant.

Proof of Prop. 3. Since we know that the state is translation invariant, we have $c_{\rho}(x)=c_{\rho}$ and $E_{\rho}(x)=E_{0}$. Furthermore, in the Coulomb case, $c_{\rho}=0$ (see Prop. 6 and Lemma 3 of Sect. 4 in [4]) which expresses the local neutrality of the state.

As we have recalled in Sect. II, this proposition will be established as soon as we have shown that:

$$
\sum_{j=1}^{n} L_{j} \rho(Q)=0 \quad \text { where } L_{j}=x_{j} \wedge \nabla_{j} .
$$

Using then Eq. (28) together with the assumption that the force is antisymmetric and radial, i.e. $x_{1} \wedge F\left(q_{1}, q_{2}\right)=-x_{2} \wedge F\left(q_{2}, q_{1}\right)$, and the fact that $E_{\rho}(x)=E_{0}$, we obtain:

$$
\begin{aligned}
k T \sum_{j=1}^{n} L_{j} \rho(Q)= & \sum_{j=1}^{n} \sigma_{\alpha_{j}} x_{j} \wedge E_{0} \rho(Q)+\int d \bar{q} \sum_{j=1}^{n} \bar{x} \wedge F\left(q_{j}, \bar{q}\right) \\
& \cdot[\rho(\bar{q}, Q)-\rho(\bar{q}) \rho(Q)] .
\end{aligned}
$$

It is also known [12] that, under the same clustering hypothesis on the correlation functions, any equilibrium state for a system of particles interacting by means of long range forces satisfying Eq. (26), (27) with $v-1 \leqq \gamma \leqq v$ will obey the following sum rule:

$$
\sum_{j=1}^{n} \sigma_{\alpha_{j}} x_{j} \rho(Q)+\int d q \sigma_{\alpha} x[\rho(q, Q)-\rho(q) \rho(Q)]=0 .
$$

In fact, this sum rule will be satisfied as soon as the clustering condition holds with $\delta>v+1$.

Combining the 1 st and $n$th equation of the BBGKY hierarchy Eq. (28), together with the sum rule (Eq. (32), the symmetry of force, i.e. $x \wedge F\left(x-x_{j}\right)=x_{j} \wedge F\left(x-x_{j}\right)$ and the definition Eq. (17) of $R(\bar{q}, q, Q)$, we can express the integrand of Eq. (31) in the following manner:

$$
\begin{aligned}
\sum_{j=1}^{n} \bar{x} & \wedge F\left(q_{j}, \bar{q}\right)[\rho(\bar{q}, Q)-\rho(\bar{q}) \rho(Q)] \\
& =-k T \bar{x} \wedge \nabla_{\bar{x}}[\rho(\bar{q}, Q)-\rho(\bar{q}) \rho(Q)]
\end{aligned}
$$




$$
\begin{aligned}
& +\sigma_{\bar{\alpha}} x \wedge E_{0}[\rho(\bar{q}, Q)-\rho(\bar{q}) \rho(Q)] \\
& +\sum_{j=1}^{n} x_{j} \wedge\left(F\left(\bar{x}-x_{j}\right)-F(\bar{x})\right) \sigma_{\bar{\alpha}} \sigma_{\alpha_{j}} \rho(\bar{q}) \rho(Q) \\
& +\int d q \sigma_{\alpha} x \wedge[F(\bar{x}-x)-F(\bar{x})] \sigma_{\bar{\alpha}} \rho(\bar{q})[\rho(q, Q) \\
& \quad-\rho(q) \rho(Q)] \\
& +\int d q \bar{x} \wedge F(\bar{q}, q) R(\bar{q}, q, Q) .
\end{aligned}
$$

To obtain the integral which appears in Eq. (31), we integrate both sides on $\bar{q}=(\bar{x}, \bar{\alpha})$ with $\bar{x}$ in a ball of radius $R$. We shall then evaluate all terms $(33 \mathrm{a}-\mathrm{e})$ in the limit $R \rightarrow \infty$.

As in Eq. (16), the gradient term (33a) is $O\left(\frac{1}{R^{\delta-v}}\right)$ by the clustering hypothesis. It thus vanishes as $R \rightarrow \infty$ as soon as $\delta>v$. The term (33b) is integrable over all space and gives, using the sum rule, Eq. (32):

$$
-E_{0} \wedge \int d \bar{q} \sigma_{\bar{\alpha}} \bar{x}[\rho(\bar{q}, Q)-\rho(\bar{q}) \rho(Q)]=E_{0} \wedge \sum_{j=1}^{n} \sigma_{\alpha_{j}} x_{j} \rho(Q) .
$$

By the assumption Eq. (30), the integrand in (33e) is integrable over $\mathbb{R}^{v} \times \mathbb{R}^{v}$ and thus, in the limit $R \rightarrow \infty$, (33e) yields:

$$
\frac{1}{2} \int d \bar{q} \int d q(\bar{x} \wedge F(\bar{q}, q)) R(\bar{q}, q, Q)=0
$$

Since $F(\bar{q}, q)$ is antisymmetric and $R(\bar{q}, q, Q)$ is symmetric in $(\bar{q}, q)$.

Using the definition of $c_{\rho}$, the integral over $\bar{q}$ of Eq. (33c) is for finite $R$

$$
\left(c_{\rho}+\rho_{B}\right) \rho(Q) \sum_{j=1}^{n} \sigma_{\alpha_{j}} x_{j} \wedge\left[\int_{|\bar{x}| \leqq R} d \bar{x}\left(F\left(\bar{x}-x_{j}\right)-F(\bar{x})\right)\right] .
$$

Using Lemma 1 and 3 of [1], the expression (35) converges in the limit $R \rightarrow \infty$ to

$$
-\left(c_{\rho}+\rho_{B}\right) \rho(Q) \sum_{j=1}^{n} \sigma_{\alpha_{j}} x_{j} \wedge\left(\mathscr{C} x_{j}\right) .
$$

$\mathscr{C}=0$ when $\gamma>v-1$, and for $\gamma=v-1 \mathscr{C}=\left\{\mathscr{C}_{r s}\right\}$ is the depolarization tensor. For a general domain $V_{0}, \mathscr{C}$ is defined by

$$
\lim _{\lambda \rightarrow \infty} \int_{V_{\lambda}} d \bar{x}\left(F_{r}(x-\bar{x})-F_{r}(-\bar{x})\right)=\sum_{s} \mathscr{C}_{r s} x^{s},
$$

where $V_{\lambda}=\left\{x \mid x=\lambda y, y \in V_{0}, \lambda>0\right\}$ is a dilatation of $V_{0}$. In particular if $V_{0}$ is a sphere, $\mathscr{C}_{r s}=\frac{\omega_{v}}{v} \delta_{r s}\left(\omega_{2}=2 \pi, \omega_{3}=4 \pi\right)$ and Eq. (36) (i.e. the contribution (33c)) gives zero.

On the other hand, before the limit $R \rightarrow \infty$, Eq. (33d), gives:

$$
\int_{|\bar{x}| \leqq R} d \bar{x} \int d q \sigma_{\alpha} x \wedge[F(\bar{x}-x)-F(\bar{x})]\left(c_{\rho}+\rho_{B}\right)[\rho(q, Q)-\rho(q) \rho(Q)] .
$$

Using the clustering condition together with Eq. (37) and the fact that $\int_{|\bar{x}| \leqq R} d \bar{x}|F(\bar{x}-x)-F(\bar{x})|=O(|\bar{x}|)$ uniformly in $R$ (see Lemmas 1 and 3 in [1]), the 
term (33d) yields, in the limit $R \rightarrow \infty$ :

$$
-\left(c_{\rho}+\rho_{B}\right) \int d q \sigma_{\alpha} x \wedge \mathscr{C} x[\rho(q, Q)-\rho(q) \rho(Q)],
$$

which will be zero by our choice of $V_{0}$.

Combining these results Eq. (33) leads to:

$$
\begin{aligned}
\sum_{j=1}^{n} \int d \bar{q} \bar{x} \wedge F(q, \bar{q})[\rho(\bar{q}, Q)-\rho(\bar{q}) \rho(Q)] \\
=E_{0} \wedge \sum_{j=1}^{n} \sigma_{\alpha_{j}} x_{j} \rho(Q)-\left(c_{\rho}+\rho_{B}\right)\left\{\sum_{j=1}^{n} \sigma_{\alpha_{j}} x_{j} \wedge \mathscr{C} x_{j} \rho(Q)\right. \\
\left.\quad+\int d q \sigma_{\alpha} x \wedge \mathscr{C} x[\rho(q, Q)-\rho(q) \rho(Q)]\right\} .
\end{aligned}
$$

Thus it follows from Eq. (31) that:

$$
\begin{aligned}
k T \sum_{j} L_{j} \rho(Q)= & -\left(c_{\rho}+\rho_{B}\right)\left\{\sum_{j=1}^{n} \sigma_{\alpha_{j}} x_{j} \wedge \mathscr{C} x_{j} \rho(Q)\right. \\
& \left.+\int d q \sigma_{\alpha} x \wedge \mathscr{C} x[\rho(q, Q)-\rho(q) \rho(Q)]\right\},
\end{aligned}
$$

i.e. $k T \sum_{j} L_{j} \rho(Q)=0$ which concludes the first part of Proposition 3 .

To prove the second part of this proposition we recall that for any periodic state of a Coulomb system $c_{\rho}=0$. Since the clustering condition $O\left(\frac{1}{|x|^{\delta}}\right)$ with $\delta>v+2$ has been used only to control the term (33d) in the limit $R \rightarrow \infty$, our proposition will remain valid when $\rho_{B}=0$, assuming that the clustering condition is satisfied with $\delta>v+1$.

Remarks. 1) The sum rule Eq. (32) is essential for the derivation of Proposition 3 and thus a clustering faster than $\frac{1}{|x|^{v+1}}$ is necessary for our proof.

2) It is conjectured that the two-dimensional jellium has a low temperature phase with orientational order. Our analysis shows that in such a phase, the clustering should decrease as or slower than $\frac{1}{|x|^{4}}$.

Corollary 1. For Jellium systems $\rho_{B} \neq 0$ and $\gamma=v-1 \geqq 1$, any equilibrium state which satisfies the clustering condition Eq. (6) with $\delta>v+2$ obeys the following sum rule:

$$
\sum_{j=1}^{n} \sigma_{\alpha_{j}} x_{j}^{r} x_{j}^{s} \rho(Q)+\int d q \sigma_{\alpha} x^{r} x^{s}(\rho(q Q)-\rho(q) \rho(Q))=\eta(Q) \delta_{r s} .
$$

Indeed, in the proof of Proposition 3, we can integrate on any sequence of volume $V_{\lambda}$ obtained by dilatation of some fixed arbitrary volume $V_{0}$. 
Using the result $K T \sum_{j} L_{j} \rho(Q)=0$ together with Eq. (39), we thus conclude that for $\gamma=v-1 \geqq 1$ and for any $V_{0}$ we must have:

$$
\rho_{B}\left\{\sum_{j=1}^{n} \sigma_{\alpha_{j}} x_{j} \wedge \mathscr{C} x_{j} \rho(Q)+\int d q \sigma_{\alpha} x \wedge \mathscr{C} x[\rho(q, Q)-\rho(q) \rho(Q)]\right\}=0 .
$$

Furthermore, the bracket in (41) vanishes if and only if $[\mathscr{C}, \xi]=0$, where:

$$
\xi^{r s}=\sum_{j=1}^{n} \sigma_{\alpha} x_{j}^{r} x_{j}^{s}+\int d q \sigma_{\alpha} x^{r} x^{s}(\rho(q, Q)-\rho(q) \rho(Q)) .
$$

Therefore, $\xi$ will commute with all symmetric matrices $\mathscr{C}$ and is thus a multiple of the identity.

Let us recall that the sum rule Eq. (40) has been established for arbitrary $\rho_{B}$ in [12] under the same clustering condition, but using an entirely different method.

Corollary 2. Equilibrium states of Coulomb systems in dimension greater than 1 can not carry an internal electric field $E_{0}$ if the clustering is $O\left(\frac{1}{|x|^{\delta}}\right)$ with $\delta>v+1$ when $\rho_{B}=0$ and with $\delta>v+2$ when $\rho_{B} \neq 0$.

This corollary follows from the rotation invariance of the state and the properties of the BBGKY equations (see Prop. 4 (iii) in [4]).

This result should be compared with the one dimensional Coulomb system which is known to have exponentially clustering states with nonvanishing internal electric field [13].

Remark. The results presented in this section will remain true for more general systems with internal degrees of freedom, such as described in Sect. II, whenever the particles interact by means of a two-body potential

$$
\Phi\left(q_{1}, q_{2}\right)=\Phi^{(S)}\left(q_{1}, q_{2}\right)+\sigma_{\omega_{1}} \sigma_{\omega_{2}} \Phi^{(L)}\left(\left|x_{1}-x_{2}\right|\right)
$$

such that the short range part of the force $F^{(S)}=-\nabla \Phi^{(S)}$ satisfies the integrability condition Eq. (7).

\section{Appendix}

We show that the contribution to (16) of space rotations vanishes, i.e.

$$
\lim _{R \rightarrow \infty} \frac{1}{2} \int_{\left|x_{1}\right| \leqq R} d q_{1} \int d q\left(L_{1}-L\right) \Phi\left(q_{1}, q\right) g\left(q_{1}, q\right)=0 .
$$

Denoting $f^{s}\left(q_{1}, q\right)=\left(x_{1}^{s}+x^{s}\right) g\left(q_{1}, q\right)$ we have

$$
\left(L_{1}-L\right)^{r} \Phi\left(q_{1}, q\right) g\left(q_{1}, q\right)=-f^{s}\left(q_{1}, q\right) F^{t}\left(q_{1}, q\right)+f^{t}\left(q_{1}, q\right) F^{s}\left(q_{1}, q\right),
$$

$(r, s, t)=$ cyclic permutation of $(1,2,3)$.

Since both terms of (A2) give a similar contribution to (A1), it is sufficient to evaluate the first one which is 


$$
\begin{aligned}
\frac{1}{2} \int_{\left|x_{1}\right| \leqq R} d q_{1} \int d q F^{t}\left(q_{1}, q\right) f^{s}\left(q_{1}, q\right)= & \frac{1}{2} \int d x \int_{\Omega} d \omega_{1} \int_{\Omega} d \omega F^{t}\left(x, \omega_{1}, \omega\right) \\
& \cdot \int_{|y| \leqq R} d y f^{s}\left(y, \omega_{1}, y-x, \omega\right) \\
= & -\frac{1}{2} \int d x \int_{\Omega} d \omega_{1} \int_{\Omega} d \omega F^{t}\left(x, \omega_{1}, \omega\right) \\
& \cdot \int_{|y| \leqq R} f^{s}\left(y+x, \omega_{1}, y, \omega\right) .
\end{aligned}
$$

We have used the antisymmetry of $F\left(q_{1}, q\right)$ and the symmetry of $f\left(q_{1}, q\right)$ under the exchange of $q_{1}$ and $q$.

Therefore, one has

$$
\begin{aligned}
\frac{1}{2}\left|\int_{\left|x_{1}\right| \leqq R} d q_{1} \int d q F^{t}\left(q_{1}, q\right) f^{s}\left(q_{1}, q\right)\right| \leqq & \frac{1}{4} \int d x \int_{\Omega} d \omega_{1} \int_{\Omega} d \omega\left|F^{t}\left(x, \omega_{1}, \omega\right)\right| \\
& \cdot \int_{\Delta_{x}} d y\left|f^{s}\left(y, \omega_{1}, y-x, \omega\right)\right|,
\end{aligned}
$$

where $\Delta_{x}=\{y|| y|\leqq R| y-x \mid, \geqq R\} \cup\{y|| y|\geqq R| y-x \mid, \leqq R\}$.

In (A3), we divide the integration on $x$ into the regions $|x| \leqq \frac{1}{2} R$ and $|x| \geqq \frac{1}{2} R$. When $|x| \leqq \frac{1}{2} R, y \in \Delta_{x}$ implies $\frac{R}{2} \leqq|y| \leqq \frac{3}{2} R$ and $\frac{3}{2} R \geqq|y-x| \geqq \frac{R}{2}$, and therefore, according to (18)

$$
\left|f^{s}\left(y, \omega_{1}, y-x, \omega\right)\right|=\left|2 y^{s}-x^{s}\right|\left|g\left(y, \omega_{1}, y-x, \omega\right)\right| \leqq \frac{M_{1}}{R^{\delta-1}}
$$

$M_{1}$ being independent of $x, y, \omega_{1}, \omega$. With this, one finds that the integral (A3) on the region $|x| \leqq \frac{1}{2} R$ is less than

$$
\begin{gathered}
\frac{1}{4} \int_{|x| \leqq R / 2} d x \int d \omega_{1} \int d \omega\left|F^{t}\left(x, \omega_{1}, \omega\right)\right| \frac{M_{1}\left|\Delta_{x}\right|}{R^{\delta-1}} \leqq \frac{M_{2}}{R^{\delta-v}} \int d x \int d \omega_{1} \\
\cdot \int d \omega|x|\left|F^{t}\left(x, \omega_{1}, \omega\right)\right|=O\left(\frac{1}{R^{\delta-v}}\right)
\end{gathered}
$$

since the volume $\left|\Delta_{x}\right|$ of $\Delta_{x}$ is $O\left(|x| R^{v-1}\right)$.

When $|x| \geqq \frac{R}{2}$, we use the fact that the correlation functions are bounded, hence

$$
f^{s}\left(y, \omega_{1}, y-x, \omega\right) \leqq c_{1}(2|y|+|x|)
$$

This implies that the integral (A3) on the region $|x| \geqq \frac{R}{2}$ is majorized by

$$
\begin{aligned}
& \frac{1}{4} \int_{|x| \geqq R / 2} d x \int d \omega_{1} \int d \omega\left|F^{t}\left(x, \omega_{1}, \omega\right)\right| \int_{\Delta_{x}} c_{1}(2|y|+|x|) d y \\
& \leqq c_{2} \int_{|x| \geqq R / 2} d x \int d \omega_{1} \int d \omega\left|F^{t}\left(x, \omega_{1}, \omega\right)\right||x|^{v+1}=o(1)
\end{aligned}
$$


since now $|y| \leqq R \leqq 2|x|$ and $\left|\Delta_{x}\right|=O\left(|x|^{v}\right)$.

This last quantity tends to zero as $R \rightarrow \infty$ by assumption. (A4) and (A5) establish that the limit (A1) vanishes.

\section{References}

1. Gruber, Ch., Martin, Ph. A.: Ann Phys. 131, 56 (1981)

2. Gruber, Ch., Lugrin, Ch., Martin, Ph. A.: Helv. Phys. Acta. 51, 829 (1979)

3. Gallavotti, G.: Nuovo Cimento 57-B, 208 (1968)

4. Gruber, Ch., Lugrin, Ch., Martin, Ph. A.: J. Stat. Phys. 22, 193 (1980)

5. Bricmont, J., Fontaine, J.-R., Lebowitz, J. L., Lieb, E. Spencer, T. Commun. Math. Phys. 78, 545 (1981)

Bricmont, J., Fontaine, J.-R., Lebowitz, J. L. Spencer, T. Commun. Math. Phys. 78, 363 (1981)

6. Fisher, M., Barber, M., Jasnow, D.: Phys. Rev. A 8, 1111 (1973)

7. Kayser, R. F., Hubbard, J.-B., Raveché, H. J.: Phys. Rev. B 24, 51 (1981)

8. Pfister, Ch. E.: Commun. Math. Phys. 79, 181 (1981)

9. Fröhlich, J., Pfister, Ch. E.: On the absence of symmetry breaking and of crystalline order in twodimensional systems, Commun. Math. Phys. 81, 277 (1981)

10. Landau, L., Fernando Perez, J., Wreszinski, W. F.: Energy gap and clustering in quantum statistical mechanics. Preprint (1981)

11. Oguey, Ch.: Invariance Euclidienne des états d'équilibre classiques. Travail de diplôme 1980 (unpublished)

12. Gruber, Ch., Lebowitz, J. L., Martin, Ph. A.: J. Chem. Phys. 75, 944 (1981)

13. Aizenman, M., Martin, Ph. A.: Commun. Math. Phys. 78, 99 (1980)

14. Martin, Ph. A.: A remark on the Goldstone theorem in statistical mechanics. To appear in Nuovo Cimento D

15. Requardt, M.: Z. Phys. B. 36, 187 (1979)

16. Requardt, M.: About the possibility of classical crystallization and poor clustering of particle distribution functions, Göttingen preprint (1981)

Communicated by E. Lieb

Received July 22, 1981 
